International Journal of Biomedicine | June 2021 - Volume 11, Issue Suppl_1: Abstracts from the Third Russian International Conference "Cryo-electron microscopy 2021: achievements and prospects"

ORAL ABSTRACT PRESENTATIONS

SESSION TITLE: STRUCTURE OF VIRUSES AND CHAPERONINS

DOI: 10.21103/IJBM.11.Suppl_1.OR9

\title{
Abstract OR-9: Cryo-EM Structure of the Reconstituted Human $\gamma$-Tubulin Ring Complex
}

\author{
Marina Serna $^{1}$, Fabian Zimmermann ${ }^{2}$, Artur Ezquerra ${ }^{1}$, \\ Rafael Fernandez-Leiro ${ }^{2}$, Jens Luders ${ }^{2}$, Oscar Llorca ${ }^{1}$ \\ ${ }^{1}$ Structural Biology Programme, Spanish National Cancer Research Centre \\ (CNIO), Madrid, Spain \\ ${ }^{2}$ Mechanisms of Disease Programme, Institute for Research in Biomedicine (IRB \\ Barcelona), Barcelona, Spain
}

Background: Microtubules (MTs) are essential cytoskeletal polymers that provide structural support for the cell and play important roles in cell division, motility, and intracellular transport. The $\gamma$-tubulin ring complex ( $\gamma \mathrm{TuRC}$ ) is the major MT nucleator in animal cells. The molecular mechanism by which the $\gamma$ TuRC promotes MT nucleation remains poorly understood although a template-based mechanism, remains the most widely accepted (Moritz et al., 2000, Kollman et al., 2010). According to this model $\gamma$ TuRC, a 2 MDa multisubunit protein complex, forms a lock washer-like structure, in which $\gamma$-tubulin molecules are arranged in a ring-shaped structure that serves as a template for the assembly of $\alpha \beta$-tubulin heterodimers.

Methods: We have set up an in vitro system to purify the human $\gamma$ TuRC using infected insect cells with recombinant baculoviruses. This complex sample was subjected to cryo-EM analysis and single-particle reconstruction.

Results: We have demonstrated that RUVBL1-RUVBL2 AAA-ATPase complex (RUVBL) controls the assembly and composition of $\gamma \mathrm{TuRC}$ in human cells both in vivo and in vitro. Likewise, RUVBL assembles $\gamma$ TuRC from a minimal set of core subunits in a heterologous co-expression system. Purified, reconstituted $\gamma$ TuRC has nucleation activity and resembles native $\gamma$ TuRC (Consolati et al., 2020, Liu et al., 2020, Wieczorek et al., 2020), as revealed by its cryo-EM structure at $\sim 4.0 \AA$ resolution. 
Conclusion: We have been able to identify novel mechanistic and structural features that determine the intricate, higher-order $\gamma \mathrm{TuRC}$ architecture (Zimmermann, Serna et al., 2020).

\section{Key Words: Cryo-EM $・$ RUVBL $\bullet \gamma$ TuRC $・$ microtubules}

*Corresponding author: Marina Serna. E-mail: mserna@cnio.es

International Journal of Biomedicine. 2021;11 Suppl 1: S10.

doi: 10.21103/IJBM.11.Suppl_1.0R9

(C)2021 International Medical Research and Development Corporation 